\title{
Laser phase noise effect and reduction in self- homodyne optical OFDM transmission system
}

\begin{abstract}
ABSTRCT
We present a laser phase noise (PN) induced effect of a phase-modulation-to-intensitymodulation conversion noise and noise pedestals underneath each of the orthogonal frequency division multiplexing (OFDM) subcarriers in a selfcoherent optical OFDM transmission using a self-homodyne technique. We provide a statistical analysis on the received symbols using a histogram to demonstrate the effect of a phase rotation term and inter-subcarrier interference individually and collectively. The PN is then compensated using a simple time delay to realign the phase walk-off of the subcarriers relative to the carrier. Significant quadrature improvements of $6.82 \mathrm{~dB}$ using $5 \mathrm{MHz}$ laser linewidth over a $720 \mathrm{~km}$ transmission length and $5.38 \mathrm{~dB}$ using 20 $\mathrm{MHz}$ over $240 \mathrm{~km}$ have been obtained with 16 quadrature amplitude modulation (QAM) over 15 GHz OFDM signal bandwidth. The technique also significantly reduced an optical-signal-tonoise ratio requirement at the bit error rate of $1 \times 10-3$ by $16.15 \mathrm{~dB}$ for 64-QAM over $160 \mathrm{~km}$. With the delay, the system can tolerate three times the chromatic dispersion-length product..
\end{abstract}

Keyword: Laser sources; Optical signals; Phase noise; Signal processing; Systems design 